MRS. SUZANNE SILVIS (Orcid ID : 0000-0001-7633-2675)

DR. MIRJAM RACHEL HELDNER (Orcid ID : 0000-0002-3594-2159)

Article type : Original Article

\title{
ANEMIA AT ADMISSION IS ASSOCIATED WITH POOR CLINICAL OUTCOME IN CEREBRAL VENOUS THROMBOSIS
}

Suzanne M. Silvis ${ }^{1}$, Elza Reinstra ${ }^{1}$, Sini Hiltunen ${ }^{2}$, Erik Lindgren ${ }^{3}$, Mirjam R. Heldner ${ }^{4}$, Maryam Mansour $^{5}$, Masoud Ghiasian ${ }^{5}$, Katarina Jood ${ }^{3}$, Susanna M. Zuurbier ${ }^{1}$, Adrien E. Groot ${ }^{1}$, Marcel Arnold $^{4}$, Miguel A. Barboza ${ }^{6}$, Antonio Arauz ${ }^{7}$, Jukka Putaala ${ }^{2}$, Turgut Tatlisumak ${ }^{2,3}$, Jonathan M. Coutinho $^{1}$, on behalf of the International CVT Consortium

${ }^{1}$ Department of Neurology, Amsterdam UMC, University of Amsterdam, Amsterdam, The Netherlands

${ }^{2}$ Department of Neurology, Helsinki University Hospital and University of Helsinki, Helsinki, Finland

3 Department of Clinical Neuroscience, Institute of Neuroscience and Physiology, Sahlgrenska Academy at University of Gothenburg, Gothenburg, Sweden and Department of Neurology, Sahlgrenska University Hospital, Gothenburg, Sweden

${ }^{4}$ Department of Neurology, Inselspital, Bern University Hospital, University of Bern, Bern, Switzerland

${ }^{5}$ Sina Hospital, Hamadan University of Medical Science, Hamadan, Iran

${ }^{6}$ Neurosciences Department, Hospital Dr. R.A. Calderón Guardia, CCSS, San José, Costa Rica

This article has been accepted for publication and undergone full peer review but has not been through the copyediting, typesetting, pagination and proofreading process, which may lead to differences between this version and the Version of Record. Please cite this article as doi: $\underline{10.1111 / \text { ene. } 14148}$

This article is protected by copyright. All rights reserved 
${ }^{7}$ National Institute of Neurology and Neurosurgery Manuel Velasco Suarez, Mexico-City, Mexico

Correspondence to: J.M. Coutinho, j.coutinho@amsterdamumc.nl, tel: +31-20-5669111

Total word count: 3309

Number of references: 18

Number of tables: 5

Number of figures: 1

Key words: cerebral venous thrombosis, anemia, prognosis

Running title: Anemia and outcome in cerebral venous thrombosis

This article is protected by copyright. All rights reserved 


\section{ABSTRACT}

Background Anemia is associated with poor clinical outcome after ischemic and hemorrhagic stroke. We examined the association between anemia and outcome in patients with cerebral venous thrombosis (CVT).

Methods Consecutive adult patients with CVT were included from seven centers. Anemia at admission was scored according to World Health Organization definitions. Poor clinical outcome was defined as a modified Rankin Scale (mRS) score 3-6 at last follow-up. A multiple imputation procedure was applied for handling missing data in the multivariable analysis. Using binary logistic regression analysis, we adjusted for age, sex, cancer, and center of recruitment (model 1). In a secondary analysis we additionally adjusted for coma, intracerebral hemorrhage, nonhemorrhagic lesion, and deep venous system thrombosis (model 2). In a sensitivity analysis we excluded patients with cancer.

Results Data of 952 patients with CVT were included, $22 \%$ of whom had anemia at admission. Patients with anemia more often had a history of cancer $(17 \%$ vs. $7 \%, p<0.001)$ than patients without anemia. Poor clinical outcome $(21 \%$ vs. $11 \%, p<0.001)$ and mortality $(11 \%$ vs. $6 \%$, $\mathrm{p}=0.07$ ) were more common among patients with anemia. After adjustment, anemia at admission increased the risk of poor outcome (adjusted odds ratio [aOR]: 2.4, 95\% confidence interval [CI] 1.5-3.7, model 1). Model 2 revealed comparable results (aOR 1.9, 95\% CI 1.2-3.2), as did the sensitivity analysis excluding patients with cancer (aOR 2.3, 95\% CI 1.3-3.8, model 1).

Conclusion The risk of poor clinical outcome is doubled in CVT patients presenting with anemia at admission.

\section{Introduction}

Cerebral venous thrombosis (CVT) is a rare thrombotic disorder and an infrequent cause of stroke that mainly affects young adults and children [1]. Approximately 9 to $27 \%$ of patients with CVT have anemia at presentation, and the presence of anemia has been shown to increase the risk of CVT $[2,3]$. In adults, the most common cause of microcytic anemia is iron deficiency. The latter has been associated with thrombocytosis and increased concentrations of factor VIII, which are risk factors for venous thrombosis [4, 5].

This article is protected by copyright. All rights reserved 
In patients with ischemic and hemorrhagic stroke, the presence of anemia had been found to be associated with poor clinical outcome $[6,7]$. One proposed mechanism is that low hemoglobin levels impair oxygen delivery to the damaged brain, and induce an inflammatory response [8]. Data from a recent single center study suggest that an association between anemia and poor clinical outcome also exists in CVT, but this study had a limited sample size [9]. The aim of our study was to assess the association between anemia and poor clinical outcome in CVT, using data from a large international CVT consortium.

\section{Methods}

Study design and patient inclusion

Data were derived from seven hospital-based CVT registries from the international CVT consortium: Amsterdam UMC (the Netherlands), Helsinki University Hospital (Finland), Sahlgrenska University Hospital (Sweden), Inselspital Bern University Hospital (Switzerland), National Institute Manuel Velasco Suarez (Mexico), Hamadan University of Medical Science (Iran), and Hospital Dr. Calderón Guardia (Costa Rica). Detailed information on consecutive patients with CVT has been collected prospectively since January 2006 (Amsterdam), January 2010 (Helsinki), January 2000 (Bern), January 2008 (Mexico-City), April 2012 (Hamadan), and May 2015 (San José). Data were collected retrospectively from January 1987 until January 2010 (Helsinki), and January 1997 (Gothenburg). Since only observational data were collected in each of the CVT registries, written informed consent was not required under applicable national laws. All data that we collected were part of routine patient care. According to local regulations, in this specific situation, no formal ethical approval is required.

We included all adult patients diagnosed with CVT until 1 March 2018. To increase the generalizability of the results, no exclusion criteria were applied. Data were recorded using a standardized case record form. Diagnosis of CVT had to be confirmed with CT-venography, MRI with MR-venography, catheter angiography, or autopsy [10]. Poor clinical outcome was defined as a score of 3 to 6 on the modified Rankin Scale (mRS), assessed at the last available follow-up contact. Mortality and mRS 0-1 were also analyzed separately.

\section{Measurement of hemoglobin and definition of anemia}

This article is protected by copyright. All rights reserved 
Hemoglobin concentration was measured in venous blood samples as a part of routine medical care, and the first hemoglobin measurement that was performed at arrival at the hospital was used for analysis, with a maximum of 48 hours after admission. We used the World Health Organization definitions for anemia: men $<130 \mathrm{~g} / \mathrm{L}$, non-pregnant women hemoglobin $<120 \mathrm{~g} / \mathrm{L}$, and pregnant women $<110 \mathrm{~g} / \mathrm{L}$ [11]. Patients with hyperhemoglobinemia (men $>175 \mathrm{~g} / \mathrm{L}$, women $>155 \mathrm{~g} / \mathrm{L}$ ) were categorized in the "no anemia" group. Anemia subgroups were defined as: mild anemia (men: 110-129 g/L, non-pregnant women: 110-119 g/L, pregnant women: 100-109 g/L), and moderate to severe anemia (men/non-pregnant women: $<110 \mathrm{~g} / \mathrm{L}$, pregnant women: $<100$ $\mathrm{g} / \mathrm{L}$ ). Anemia was further categorized as microcytic (mean corpuscular volume $[\mathrm{MCV}]<80 \mathrm{fL}$ ), normocytic (MCV 80-100 fL), or macrocytic (MCV >100 fL).

\section{Statistical analysis}

We compared patients with anemia to those without anemia. Differences between groups were analyzed with a chi-square test, Fisher's exact test, or Mann-Whitney U-test, as appropriate. A multiple imputation procedure was used to account for handling missing data in the multivariable analysis. The following variables were imputed: hemoglobin concentration, $\mathrm{MCV}$, thrombocytes, baseline coma, intracerebral hemorrhage, non-hemorrhagic lesion (cerebral edema/infarction), deep venous system thrombosis, and mRS at follow-up. The proportion of patients with missing data prior to imputation is reported and for baseline characteristics only non-imputed data are shown. In total, 5 data sets were imputed, and results were pooled according to Rubin's rules. We applied multivariable binary logistic regression analysis to study the association between admission anemia and clinical outcome (mRS 0-2 vs. 3-6, mRS 0-1 vs. 2-6, and mortality), using two different models. In the first model we adjusted for potential confounders that were considered to have a causal relation both with anemia and outcome: age, sex, and cancer, and for center of recruitment. In the second model, we used all variables of model 1 and additionally adjusted for known predictors of poor outcome in CVT: coma, intracerebral hemorrhage, nonhemorrhagic lesion, and deep venous system thrombosis [3]. We performed a sensitivity analysis excluding patients with cancer. We also used multivariable ordinal logistic regression analysis to calculate the adjusted common odds ratio (acOR) for a shift in the direction of poor clinical outcome on the mRS in the presence of anemia. In a separate analysis, we analyzed hemoglobin as a continuous variable, and in subgroup analyses we stratified for mild versus moderate or severe anemia. Further, we performed subgroup analysis in men and women, women who were pregnant 
or postpartum, women using oral contraceptives, and in CVT patients with cancer. All data were analyzed with SPSS statistical software, version 24.

\section{Results}

There were 952 patients diagnosed with CVT within the study period ( $\mathrm{n}=225$ Amsterdam cohort, $\mathrm{n}=246$ Helsinki cohort, $\mathrm{n}=127$ Gothenburg cohort, $\mathrm{n}=182$ Bern cohort, $\mathrm{n}=77$ Mexico-City cohort, $n=70$ Hamadan cohort, $n=25$ San-José cohort). Numbers of patients with imputed data were as follows: hemoglobin $n=78, \operatorname{MCV} n=238$, thrombocytes $n=88$, baseline coma $n=4$, intracerebral hemorrhage $n=6$, non-hemorrhagic lesion $(n=5)$, deep venous system thrombosis $n=1, m R S$ at last follow up $n=2$. After exclusion of the 78 patients with missing hemoglobin, 874 patients were included in the baseline comparison (Table 1). There were no significant differences in the baseline characteristics between the included and excluded patients (data not shown).

Of the 874 included patients, 196 (22\%) had anemia (median hemoglobin $109 \mathrm{~g} / \mathrm{L}$, interquartile range [IQR] 94-117). Of the patients with anemia, 102 patients (52\%) had mild anemia and 94 $(48 \%)$ had moderate to severe anemia. There were 56 patients $(29 \%)$ with microcytic anemia, 98 patients (50\%) with normocytic anemia, and 2 patients (1\%) with macrocytic anemia. Hyperhemoglobinemia was present in 42 patients. Causes of anemia are reported in Table 2. Hemoglobin values normalized in 10 patients during admission, one of whom received a blood transfusion. In total, 26/196 patients (13\%) received a blood transfusion during admission. Patients with admission anemia were younger (median age 37 vs. 42 years, $p=0.02$ ) more often had a history of cancer $(17 \%$ vs. $7 \%, p<0.001)$, more often presented with coma $(11 \%$ vs. $5 \%, p=0.001)$, and more often had non-hemorrhagic parenchymal lesions (44\% vs. 30\%, p<0.001, Table 1).

Median duration of follow-up was 6 months in patients with anemia (IQR 5-13 months) and 7 months in patients without anemia (IQR 6-12 month, $\mathrm{p}=0.276$ ). Poor clinical outcome was more common in patients with anemia (mRS 3-6: $21 \%$ vs. $11 \%, p<0.001$ ). Mortality was also increased in anemic patients $(11 \%$ vs. $6 \% ; p=0.07)$. After adjustment, anemia at baseline was associated with an increased risk of poor clinical outcome (adjusted odds ratio [aOR] mRS 3-6: 2.4, 95\% CI 1.5-3.7, model 1; Table 3). There was also a trend towards an increased risk of mortality (aOR 1.7, 95\% CI 0.9-3.1) and a lower chance of mRS 0-1 (aOR 0.7, 95\% CI 0.5-1.0). Additional adjustment for coma, intracerebral hemorrhage, non-hemorrhagic lesion, and deep venous system thrombosis, 
revealed similar results (aOR 1.9, 95\% CI 1.2-3.2; model 2). The results of the sensitivity analysis excluding patients with cancer were comparable to the main analysis (aOR 2.3, 95\% CI 1.3-3.8).

The full distribution of the $\mathrm{mRS}$ is shown in figure 1. Ordinal logistic regression analysis demonstrated a shift in the distribution of the mRS towards poor clinical outcome in the presence of anemia (adjusted common odds ratio 1.4, 95\% CI 1.0-1.9). When hemoglobin was analyzed as a continuous variable, there was an inverse association between hemoglobin and poor clinical outcome (aOR for mRS 3-6 per $10 \mathrm{~g} / \mathrm{L}$ increase in hemoglobin concentration: $0.83,95 \%$ CI $0.72-$ $0.95)$.

Stratification by severity of anemia revealed that the risk of poor clinical outcome was increased in both patients with mild and patients with moderate to severe anemia (aOR mild anemia 1.8, 95\% CI 1.0-3.3; aOR moderate to severe anemia 3.1, 95\% CI 1.8-5.5; Table 4). Subgroup analyses demonstrated an increased risk of poor clinical outcome in both male and female anemic patients with CVT (Table 3).

\section{Discussion}

In this large international observational study, we found that anemia was an independent predictor of poor clinical outcome in patients with CVT. The risk of death or dependency (mRS 3-6) was approximately doubled in patients with anemia. Furthermore, we observed a meaningful sign of exposure-response relationship.

The strength of the association between anemia and poor clinical outcome in our CVT cohort is similar to results of studies in arterial ischemic stroke and hemorrhagic stroke [6]. Our study may have been underpowered to detect an association with mortality. Studies on ischemic stroke had data of thousands of patients available and the rate of mortality is also higher is this condition. We identified only one other study that examined the association between outcome and anemia in CVT [9]. This study found a more pronounced association between anemia and both poor clinical outcome (aOR 3.6) and mortality (aOR 5.5) than the current study. However, this was a retrospective, single center study and thus the estimate of the strength of the association may be less accurate. Interestingly, in this study, mortality was twice as high compared to our study (14\% vs. $7 \%$ ). This rate of mortality is also higher than what is generally reported in the literature on 
CVT [12]. There were differences in baseline characteristics that may partly explain the difference in mortality. Most notably, the proportion of anemic patients that was comatose at admission was substantially higher (25 vs. $11 \%$ ) and coma is one of the strongest predictors of poor outcome in CVT [3]. Also, a very large proportion of patients in the study by Liu et al. received endovascular treatment, which may also indicate a generally more severe clinical condition of their population.

There are several hypotheses that may explain the association between anemia and poor clinical outcome in patients with arterial ischemic stroke, and some of these may also apply to CVT. Anemia is thought to induce a hyperdynamic circulation that triggers an inflammatory response, consequently leading to increased thrombus formation [8]. In the presence of a parenchymal lesion, lower oxygen carrying capacity of the blood in anemic patients may induce increased hypoxia in the affected tissue $[6,13,14]$. Further, there is evidence from experimental studies that hypoxic anemia can lead to secondary ischemic brain tissue due to upregulation of inflammatory mediators $[15,16]$. The higher rate of baseline parenchymal lesions in anemic patients provides support to this hypothesis.

Despite the abovementioned hypotheses, the observation of an association obviously does not prove the presence of a causal relationship between anemia and outcome in CVT. However, if such a causal relationship would exist, treatment to raise hemoglobin levels in anemic patients, might improve outcome. The efficacy of red blood cell transfusion has been evaluated in one observational study in anemic patients with intracerebral hemorrhage. The results were promising, but until these results have been confirmed in a randomized trial, a more liberal transfusion practice outside the current transfusion guidelines in anemic CVT patients is not justified [17, 18].

Strengths of our study are the large sample size and the multi-center design with data from both middle and high-income countries. Our study also has several limitations. First, we had to exclude 78 cases $(8 \%)$ for baseline analysis because of missing baseline hemoglobin. In order to minimize the risk of bias, we used a multiple imputation procedure to account for the missing data in multivariable analysis. Second, although data from consecutive cases were included, part of our data was collected retrospectively. Third, there was no pre-defined follow-up time point. Centers followed local protocols regarding follow-up intervals and last available mRS was used in analysis. However, median duration of follow-up was not different between patients with and 
without anemia, and we adjusted the analysis for center of recruitment, which negates this potential bias. Fourth, analysis by red blood cell morphology to evaluate anemia was not possible, because these ancillary investigations were not routinely performed. Finally, our study was underpowered to reliably investigate the risk of poor clinical outcome in specific CVT subgroups.

In conclusion, our study shows that admission anemia occurs in about one fifth of patients with CVT and that anemia is an independent predictor of poor clinical outcome in these patients. Whether a causal relationship underlies this association and whether increasing hemoglobin levels improves clinical outcome require further study.

\section{Acknowledgement}

This paper was not supported by any funding.

\section{Disclosure of conflicts of interest}

J.P. reports personal fees from Boehringer-Ingelheim, during the conduct of the study. T.T. reports grants from Helsinki University Central Hospital, grants from University of Gothenburg, grants from Sahlgrenska University Hospital, during the conduct of the study; grants and personal fees from Boehringer Ingelheim, personal fees from Lumosa Pharm, grants and personal fees from Bayer, personal fees from BMS, outside the submitted work. In addition, T.T. has a patent use of a mast cell activation or degranulation blocking agent in the manufacture of a medicament for the treatment of a patient subjected to thrombolyses. Patent no: US8163734. Filed: February 13, 2004. Issued: April 24, 2012. J.M.C. has received research grants for CVT from two non-profit organizations: the Dutch Thrombosis Society and the Netherlands Brain Foundation, and reports fees from Boehringer Ingelheim and Bayer. All fees paid to institute and used to fund scientific research. The other authors declare no financial or other conflicts of interest.

This article is protected by copyright. All rights reserved 
1. Silvis SM, de Sousa DA, Ferro JM, Coutinho JM. Cerebral venous thrombosis. Nat Rev Neurol. 2017;13:555-65.

2. Coutinho JM, Zuurbier SM, Gaartman AE, et al. Association Between Anemia and Cerebral Venous Thrombosis: Case-Control Study. Stroke. 2015;46:2735-40.

3. Ferro JM, Canhao P, Stam J, et al. Prognosis of cerebral vein and dural sinus thrombosis: results of the International Study on Cerebral Vein and Dural Sinus Thrombosis (ISCVT). Stroke. 2004;35:664-70.

4. Ho KM, Yip CB, Duff O. Reactive thrombocytosis and risk of subsequent venous thromboembolism: a cohort study. J Thromb Haemost. 2012;10:1768-74.

5. Livesey JA, Manning RA, Meek JH, et al. Low serum iron levels are associated with elevated plasma levels of coagulation factor VIII and pulmonary emboli/deep venous thromboses in replicate cohorts of patients with hereditary haemorrhagic telangiectasia. Thorax. 2012;67:328-33.

6. Barlas RS, Honney K, Loke YK, et al. Impact of Hemoglobin Levels and Anemia on Mortality in Acute Stroke: Analysis of UK Regional Registry Data, Systematic Review, and Meta-Analysis. J Am Heart Assoc. 2016;5.

7. Faller N, Limacher A, Mean M, et al. Predictors and Causes of Long-Term Mortality in Elderly Patients with Acute Venous Thromboembolism: A Prospective Cohort Study. Am J Med. 2017;130:198206.

8. Kaiafa G, Savopoulos C, Kanellos I, et al. Anemia and stroke: Where do we stand? Acta Neurol Scand. 2017;135:596-602.

9. Liu K, Song B, Gao Y, et al. Long-Term Outcomes in Patients with Anemia And Cerebral Venous Thrombosis. Neurocrit Care. 2018;10.1007/s12028-018-0544-6.

10. Saposnik G, Barinagarrementeria F, Brown RD, Jr., et al. Diagnosis and management of cerebral venous thrombosis: a statement for healthcare professionals from the American Heart Association/American Stroke Association. Stroke. 2011;42:1158-92.

11. World Health Organization. Haemoglobin concentrations for the diagnosis of anaemia and assessment of severity. 2011 [Available from: http://www.who.int/vmnis/indicators/haemoglobin.pdf.

12. Coutinho JM, Zuurbier SM, Stam J. Declining mortality in cerebral venous thrombosis: a systematic review. Stroke. 2014;45:1338-41.

13. Shao J, Xi G, Hua Y, Schallert T, Felt B. Intracerebral hemorrhage in the iron-deficient rat. Stroke. 2005;36:660-4.

14. Bellwald $S$, Balasubramaniam R, Nagler M, et al. Association of anemia and hemoglobin decrease during acute stroke treatment with infarct growth and clinical outcome. PLoS One. 2018;13:e0203535.

This article is protected by copyright. All rights reserved 
15. McLaren AT, Marsden PA, Mazer CD, et al. Increased expression of HIF-1alpha, nNOS, and VEGF in the cerebral cortex of anemic rats. Am J Physiol Regul Integr Comp Physiol. 2007;292:R403-14.

16. Moro MA, Cardenas A, Hurtado O, Leza JC, Lizasoain I. Role of nitric oxide after brain ischaemia. Cell Calcium. 2004;36:265-75.

17. Sheth KN, Gilson AJ, Chang Y, et al. Packed red blood cell transfusion and decreased mortality in intracerebral hemorrhage. Neurosurgery. 2011;68:1286-92.

18. Carson JL, Stanworth SJ, Roubinian N, et al. Transfusion thresholds and other strategies for guiding allogeneic red blood cell transfusion. Cochrane Database Syst Rev. 2016;10:CD002042.

Table 1 Baseline characteristics and treatment

$\begin{array}{lll}\text { Anemia } & \text { No anemia } & \text { P-value } \\ \mathrm{N}=196 & \mathrm{~N}=678 & \end{array}$

\section{Demographics}

Women, $\mathrm{n}, \%$ $144 / 196(74 \%) \quad 464 / 678(68 \%) \quad 0.17$

Median age [IQR] $38(27-49)$

$42(29-54)$

0.02

Onset to diagnosis (median [IQR] in days)

$5(2-10)$

0.25

\section{Risk factors: $\mathbf{n} / \mathrm{N}$ [\%]}

Oral contraceptive use $\mathrm{a}^{\mathrm{a}}$

Pregnancy, puerperium ${ }^{\mathrm{a}}$

$\begin{array}{lll}57 / 142(40 \%) & 236 / 461(51 \%) & 0.02 \\ 25 / 144(17 \%) & 46 / 464(10 \%) & 0.02\end{array}$

Previous thrombosis

$15 / 196(8 \%)$

63/671 (9\%)

0.46

Cancer

$33 / 196(17 \%)$

$50 / 677(7 \%)$

$<0.001$

\section{Characteristics at presentation}

Headache

Focal neurological deficits

$\begin{array}{lll}158 / 192(82 \%) & 587 / 672(87 \%) & 0.07 \\ 126 / 193(65 \%) & 391 / 676(58 \%) & 0.06 \\ 74 / 195(38 \%) & 202 / 673(30 \%) & 0.04 \\ 21 / 196(11 \%) & 31 / 677(5 \%) & 0.001\end{array}$

Coma $(\mathrm{GCS}<9)$

$21 / 196(11 \%)$

$31 / 677(5 \%)$

0.001

This article is protected by copyright. All rights reserved 
Laboratory findings ${ }^{b}$

Glucose (mmol/l)

$6.3 \pm 1.7$

$6.5 \pm 2.4$

0.57

Mean corpuscular volume (fl)

$81 \pm 12$

$89 \pm 5$

$<0.001$

Thrombocyte count $\left(10^{\wedge} 9 / 1\right)$

$283 \pm 117$

$260 \pm 83$

0.28

\section{Radiological characteristics}

Any parenchymal lesion

$118 / 194(61 \%) \quad 348 / 677(51 \%) \quad 0.02$

Non-hemorrhagic lesion

$85 / 194(44 \%) \quad 205 / 677(30 \%) \quad<0.001$

Intracranial hemorrhage

69/193 (36\%) $\quad 213 / 677(32 \%) \quad 0.26$

Superior sagittal sinus thrombosis

$95 / 195(49 \%) \quad 361 / 678(53 \%) \quad 0.27$

Deep venous system thrombosis

$20 / 195(10 \%) \quad 72 / 678(11 \%) \quad 0.88$

Thrombosis in multiple veins or sinus $(\geq 3)$

$32 / 195(16 \%) \quad 165 / 178(24 \%) \quad 0.02$

\section{Treatment}

Anticoagulation

$186 / 196(95 \%) \quad 654 / 677(97 \%) \quad 0.27$

Endovascular treatment

$15 / 196(8 \%) \quad 37 / 678(6 \%) \quad 0.25$

Decompressive hemicraniectomy

17/196 (9\%)

$33 / 678(5 \%)$

0.04

$I Q R$ interquartile range, GCS Glasgow Coma Scale

a Percentage of women

b Mean (+/- SD)

This article is protected by copyright. All rights reserved 
Table 2 Cause of anemia

\begin{tabular}{|l|l|}
\hline Suspected cause & Frequency n/N (\%) \\
\hline Iron deficiency & $43 / 196(22 \%)$ \\
\hline Cancer & $28 / 196(14 \%)$ \\
\hline $\begin{array}{l}\text { Hematological condition } \\
\text { other than cancer }\end{array}$ & $16 / 196(8 \%)$ \\
\hline $\begin{array}{l}\text { Inflammatory bowel disease } \\
\text { or related gastro-intestinal } \\
\text { condition }\end{array}$ & $15 / 196(8 \%)$ \\
\hline Pregnancy/puerperium & $20 / 196(10 \%)$ \\
\hline Infection & $10 / 196(5 \%)$ \\
\hline Other & $15 / 196(8 \%)$ \\
\hline Unknown & $49 / 196(25 \%)$ \\
\hline
\end{tabular}

This article is protected by copyright. All rights reserved 
Table 3 Association between anemia and clinical outcome

\begin{tabular}{llllll}
\hline & Anemia & No anemia & $\begin{array}{l}\text { Unadjusted } \\
\text { OR, 95\% CI }\end{array}$ & $\begin{array}{l}\text { Adjusted } \\
\text { OR }^{\mathrm{a}} 95 \% \text { CI }\end{array}$ & $\begin{array}{l}\text { Adjusted } \\
\text { OR }^{\mathrm{b}} 95 \% \\
\text { CI }\end{array}$ \\
& & & & & \\
\hline mRS 3-6 & $47 / 219(21 \%)$ & $82 / 733(11 \%)$ & $2.2(1.4-3.4)$ & $2.4(1.5-3.7)$ & $1.9(1.2-3.2)$ \\
Mortality & $23 / 219(11 \%)$ & $44 / 733(6 \%)$ & $1.9(1.0-3.5)$ & $1.7(0.9-3.1)$ & $1.4(0.7-2.9)$ \\
mRS 0-1 & $124 / 219(57 \%)$ & $476 / 733(65 \%)$ & $0.7(0.5-1.0)$ & $0.7(0.5-1.0)$ & $0.8(0.6-1.2)$
\end{tabular}

OR, odds ratio; CI, confidence interval; mRS, modified Rankin Scale

Binary logistic regression analysis is based on the pooled estimate after multiple imputation to account for missing variables.

aAdjusted for age, sex, cancer, and center of recruitment

${ }^{b}$ Adjusted for sex, age, cancer, coma, intracerebral hemorrhage, non-hemorrhagic lesion, thrombosis deep venous system, and center of recruitment. 
Table 4 Stratification by severity of anemia

$\begin{array}{llll}\text { No. of patients } & \text { Unadjusted } & \text { Adjusted } & \text { Adjusted } \\ \text { OR, 95\% CI } & \text { OR }^{\mathrm{a}} 95 \% \mathrm{CI} & \mathrm{OR}^{\mathrm{b}} 95 \% \\ & & \mathrm{CI}\end{array}$

Functional status mRS, 0-2 mRS, 3-6

Mild $\quad 95 / 823(12 \%) \quad 21 / 129(16 \%) \quad 1.8(1.0-3.2) \quad 1.8(1.0-3.3) \quad 1.5(0.8-2.8)$

Moderate to $\quad 77 / 823(9 \%) \quad 27 / 129(21 \%) \quad 2.6(1.5-4.7) \quad 3.1(1.8-5.5) \quad 2.4(1.2-4.7)$

severe

Mortality $\quad$ Alive $\quad$ Dead

Mild $\quad 106 / 885(12 \%) \quad 10 / 67(15 \%) \quad 1.6(0.7-3.6) \quad 1.4(0.6-3.1) \quad 1.3(0.5-3.1)$

Moderate to $\quad 91 / 885(10 \%) \quad 13 / 67(19 \%) \quad 2.1(1.0-4.4) \quad 2.2(1.0-4.7) \quad 1.6(0.6-4.0)$

severe

OR, odds ratio; CI, confidence interval; mRS, modified Rankin Scale

Binary logistic regression analysis based on the pooled estimate after multiple

imputation to account for missing variables.

${ }^{a}$ Adjusted for age, sex, cancer, and center of recruitment

${ }^{\mathrm{b}}$ Adjusted for sex, age, cancer, coma, intracerebral hemorrhage, non-hemorrhagic

This article is protected by copyright. All rights reserved 
lesion, thrombosis deep venous system, and center of recruitment.

Table 5 Outcome (mRS 3-6) in specific subgroups in the presence of anemia

\begin{tabular}{lllll} 
& Anemia & No anemia & $\begin{array}{l}\text { Unadjusted } \\
\text { OR, 95\% CI }\end{array}$ & $\begin{array}{l}\text { Adjusted OR } \\
\text { 95\% CI }\end{array}$ \\
& mRS 3-6 & mRS 3-6 & & \\
\hline Men & $15 / 58(26 \%)$ & $27 / 236(11 \%)$ & $2.5(1.2-5.0)$ & $2.7(1.2-5.7)^{\mathrm{a}}$ \\
Women & $32 / 158(20 \%)$ & $54 / 498(11 \%)$ & $2.1(1.2-3.6)$ & $2.3(1.3-4.1)^{\mathrm{a}}$ \\
Pregnancy/puerperium & $6 / 26(23 \%)$ & $4 / 49(8 \%)$ & $2.6(0.7-9.6)$ & $2.5(0.6-10.3)^{\mathrm{a}}$ \\
Oral contraceptive use & $11 / 65(17 \%)$ & $12 / 253(5 \%)$ & $4.2(1.6-10.8)$ & $2.6(0.9-7.8)^{\mathrm{a}}$ \\
Cancer & $14 / 36(39 \%)$ & $15 / 54(28 \%)$ & $1.8(0.6-5.0)$ & $2.8(0.9-9.4)^{\mathrm{b}}$ \\
\hline
\end{tabular}

OR, odds ratio; CI, confidence interval; mRS, modified Rankin Scale

Binary logistic regression analysis based on the pooled estimate after multiple imputation to account for missing variables.

${ }^{a}$ Adjusted for age, cancer, and center of recruitment

${ }^{\mathrm{b}}$ Adjusted for age, sex, and center of recruitment

This article is protected by copyright. All rights reserved 


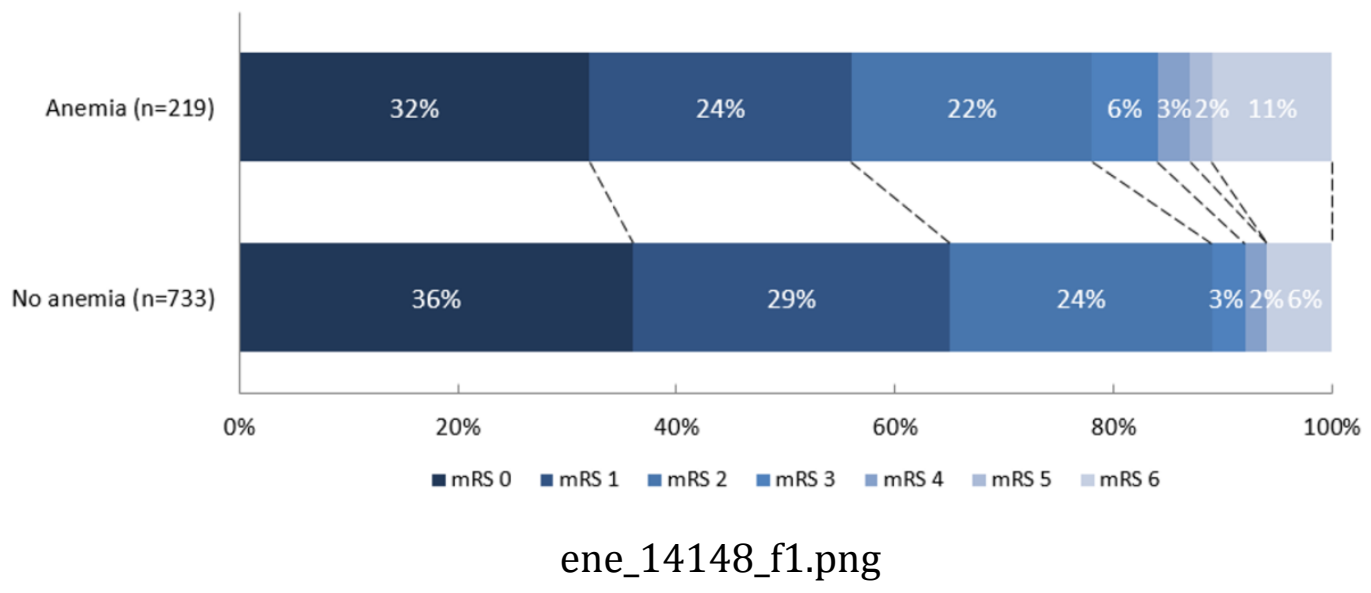

This article is protected by copyright. All rights reserved 